\title{
Effect of Labor Augmentation Using Oxytocin on Neonatal Outcome, A Case Control Study
}

\author{
Farag $\mathrm{A}^{1 *}$, Ibrahim $\mathrm{H}^{2}$ and Elnaggar $\mathrm{A}^{1}$ \\ ${ }^{1}$ Department of Obstetrics and Gynecology, Ain Shams University, Egypt \\ ${ }^{2}$ Research Fellow, Ain Shams University Maternity Hospital, Egypt
}

Submission: December 30, 2019; Published: January 08, 2020

*Corresponding author: Amr Hassan Farag, Department of Obstetrics and Gynecology, Ain Shams University, Egypt

\begin{abstract}
Aims: To re-evaluate whether augmentation of labor with oxytocin in dysfunctional labor, has a hazardous effect on neonatal Apgar scores, umbilical cord $\mathrm{pH}$ or blood gases.

Materials and Methods: Prospective study where one hundred and fifty-three (153) patients in spontaneous labor were involved in the analysis. Seventy-nine patients (Group I) with hypo-contractile uterine activity were managed by low-dose oxytocin protocol for labor augmentation, while 74 patients (Group II) were not managed by augmentation of labor using oxytocin. Primary outcomes were Apgar score at 5th minutes of birth and umbilical artery pH, while secondary outcomes were Apgar score at 1st minute, arterial p02, pCO2 and base deficit of all neonates.

Result: In this study, there was no significant difference between cases and controls regarding; Apgar score at 1st minute, Apgar score at 5th minute, mean umbilical artery pCO2 and pO2 while mean umbilical artery pH was significantly lower and umbilical artery base deficit was significantly higher in the case group compared to the control group. Moreover, umbilical artery $\mathrm{pH}$ was negatively correlated to duration of oxytocin administration.
\end{abstract}

Conclusion: Oxytocin use to augment labor, although affects umbilical cord blood gases, doesn't significantly affect neonatal outcome if used in a proper dose to produce efficient uterine contractions

Keywords: Apgar score; Augmentation; Cord gases; Oxytocin

\section{Introduction}

Approximately 20 percent of labors involve either protraction or arrest disorders [1]. The active management of labor was introduced during the 1960's at the National Maternity Hospital in Dublin, Ireland to augment labor in nulliparous women. Proponents of this approach have suggested that it also safely reduces the number of Cesarean deliveries [2]. Oxytocin increases frequency of contractions, causes initial transient increase in the base tone (incomplete relaxation) and increases the amplitude and duration of phasic contractions [3]. During parturition, prolonged tonic contractions might be harmful for fetuses because an increase of the intrauterine pressure would disturb the feto-maternal circulation and may occlude blood vessels within the myometrium [4]. Utero-placental hypo-perfusion is the major cause of both respiratory and metabolic acidemia, with progression from the former to the latter over time if decreased utero-placental blood flow is not corrected [5], hence various studies were designed to study the safety of using oxytocin infusion during the course of labor on neonatal clinical and acid-base condition at delivery and the likelihood of causing perinatal asphyxia.

\section{Materials and Methods}

The study was conducted in Ain Shams University Maternity Hospital and El-Sahel Teaching Hospital in Cairo, Egypt, which are two big tertiary hospitals, from January till October 2010, where 180 patients in spontaneous labor were initially enrolled in this case-control study after informed consent and approval of the study protocol by the institute ethics committee. Ninety patients who fulfilled the selection criteria, were diagnosed as primary failure of progression in labor due to hypo-contractile uterine activity and managed by augmentation of labor using low-dose oxytocin (Syntocinon ${ }^{\circledR} 10 \mathrm{IU} / \mathrm{ml}$ concentrate for solution for 
infusion) (Group I). Another group of ninety patients also fulfilled the selection criteria but were not managed by augmentation of labor using oxytocin (Group II). Participants were all aged between 18 and 45 years old, free of medical disorders, planned for vaginal delivery having singleton pregnancy at full term admitted at $3-5 \mathrm{~cm}$ cervical dilatation with vertex presentation of estimated fetal weight between 2500 to 4000 grams with average amniotic fluid volume and morphologically normal fetus, with no evidence of fetal distress or placental insufficiency and with uncomplicated pregnancy as detected by antenatal course.

All neonates were assessed using Apgar score at $1^{\text {st }}$ and $5^{\text {th }}$ minutes by same observer and umbilical cord blood samples were obtained from all neonates where a segment of $10-20 \mathrm{~cm}$ of umbilical cord was doubly clamped and excised immediately after delivery then $1-2 \mathrm{ml}$ blood withdrawn in a heparinized syringe; one from the artery and the other from the vein then kept in ice and transported to the laboratory within 60 minutes and analyzed using blood analyzer (i-STAT300F, Abbott Point of Care Inc., USA).

Prior to start of the study, all participants were assessed to confirm eligibility then were consistently managed according to the local institutional intra-partum protocol and received proper monitoring and supportive care. Normal FHR pattern and assessment of uterine activity were confirmed using Cardiotocography (CTG) for 30 minutes before starting interventions. Intermittent auscultation was used in low-risk women while continuous electronic fetal monitoring was used for the high-risk cases. Vaginal examination was performed two hourlies, unless clinically indicated, to monitor progress of labor. Artificial rupture of fetal membranes (ROM) was considered, in women with intact membranes, if there was poor progress of labor (cervical dilatation $<1 \mathrm{~cm} /$ hour) with inefficient uterine contractions 2 hours after ROM, the woman was assigned to Group I, receiving low-dose oxytocin protocol using oxytocin dilution of $10 \mathrm{mU} / \mathrm{mL}$, initial dose of $2 \mathrm{mU} / \mathrm{min}(12 \mathrm{~mL} / \mathrm{hr})$, and incremental increase of $2 \mathrm{mU}(12 \mathrm{~mL}$ ) every 45 minutes until adequate contractions with the maximum dose being $16 \mathrm{mU} / \mathrm{min}(96 \mathrm{~mL} /$ hr) [6]. Interpretation of intrapartum fetal heart rate pattern and uterine contractions was done according to ACOG guidelines $[7,8]$. Cases of uterine hyper-stimulation, antepartum hemorrhage, cases needed instrumental delivery or emergency Cesarean section, as well as neonates with apparent congenital anomalies at birth were all excluded (11 from group I and 16 from group II) and not involved in the analysis. The primary outcomes considered were Apgar score at $5^{\text {th }}$ minute of birth and umbilical artery $\mathrm{pH}$, while secondary outcomes were Apgar score at $1^{\text {st }}$ minute of birth, umbilical artery p02, pCO2 and base excess of all neonates.

\section{Statistical Analysis}

Statistical analysis was done on a personal computer using IBM $®$ SPSS $®$ Statistics version 19 (IBM $®$ Corporation, Armonk, NY, USA). Kolmogorov-Smirnov test of normality was applied to all measured variables where quantitative variables were described as mean and standard deviation (SD), number and percentage, or median and inter-quartile range (IQR) as appropriate. The independent sample t-test was used to compare the two groups as regards quantitative variables in parametric data while Mann Whitney U test was used for non-parametric data and Chi square test was used for qualitative data. Relative risk and Odds ratio were used to calculate likelihood of negative effect with a confidence interval of 95\%. Pearson's correlation was used for measuring correlation between different variables. Significance level was always set at 0.05 .

\section{Result}

Statistical analysis included 153 patients; 79 in Group I and 74 in Group II. Table 1 shows no significant difference between cases and controls regarding mean maternal age, BMI, gestational age, parity and mean cervical dilatation at enrollment $(P>0.05)$.

Table 1: Difference in demographic data between Groups.

\begin{tabular}{|c|c|c|c|}
\hline \multirow{2}{*}{ Studied Parameters } & \multicolumn{2}{|c|}{ Studied Groups } \\
\cline { 2 - 4 } & $\begin{array}{c}\text { Group I] } \\
\text { (cases) } \\
(\mathbf{n = 7 9 )}\end{array}$ & $\begin{array}{c}\text { Group II } \\
\text { (controls) } \\
\text { (n=74) }\end{array}$ & P Value \\
\hline Maternal age (years) & $27.04 \pm 4.64$ & $28.27 \pm 6.78$ & 0.19 \\
\hline BMI (Kg/m $\left.\mathrm{m}^{2}\right)$ & $24.91 \pm 2.32$ & $25.38 \pm 3.43$ & 0.32 \\
\hline Gestational age (weeks) & $39.20 \pm 1.40$ & $39.38 \pm 1.31$ & 0.414 \\
\hline Parity & $0(0-2)$ & $0(0-3)$ & 0.121 \\
\hline Baseline cervical dilatation (cm) & $4.4 \pm 0.5$ & $4.5 \pm 0.2$ & 0.111 \\
\hline
\end{tabular}

BMI body mass index [calculated as weight $(\mathrm{kg})$ divided by squared height $\left(\mathrm{m}^{2}\right)$ ]

$\mathrm{Kg}$, Kilograms; $\mathrm{m}^{2}$, square meters; $\mathrm{cm}$, centimeters.

Analysis using independent student's t-test or Mann Whitney $U$ test as appropriate

Data are presented as mean \pm standard deviation or median (interquartile range, IQR) as appropriate. 


\section{Journal of Gynecology and Women's Health}

Table 2: Difference between Groups regarding studied neonatal outcome data.

\begin{tabular}{|c|c|c|c|}
\hline \multirow[b]{2}{*}{ Studied Parameters } & \multicolumn{2}{|c|}{ Studied Groups } & \multirow[b]{2}{*}{ P Value } \\
\hline & $\begin{array}{l}\text { Group I' } \\
\text { (cases) } \\
\text { (n=79) }\end{array}$ & $\begin{array}{c}\text { Group II } \\
\text { (controls) } \\
(n=74)\end{array}$ & \\
\hline Apgar score at $1^{\text {st }}$ minute & $8(7-9)$ & $9(8-9)$ & 0.484 \\
\hline Apgar score at $5^{\text {th }}$ minute & $9(9-9)$ & $9(9-9)$ & 0.897 \\
\hline Umbilical vein $\mathrm{pH}$ & $7.34 \pm 0.04$ & $7.35 \pm 0.05$ & 0.173 \\
\hline Umbilical artery $\mathrm{pH}$ & $7.22 \pm 0.08$ & $7.25 \pm 0.07$ & $0.015 \pi$ \\
\hline Umbilical artery base deficit (mmol/L) & $5.65 \pm 3.39$ & $4.50 \pm 3.02$ & $0.029 \pi$ \\
\hline Umbilical artery $\mathrm{pO}_{2}(\mathrm{mmHg})$ & $15.60 \pm 2.53$ & $15.51 \pm 2.75$ & 0.833 \\
\hline Umbilical artery $\mathrm{pCO}_{2}(\mathrm{mmHg})$ & $54.03 \pm 6.97$ & $51.98 \pm 6.99$ & 0.0714 \\
\hline Neonatal birth weight (grams) & $3178.48 \pm 376.10$ & $3204.73 \pm 341.45$ & 0.653 \\
\hline Neonatal sex Male no. (\%) & $33(41.77 \%)$ & $36(48.65 \%)$ & \\
\hline Female no. (\%) & $46(58.23 \%)$ & $38(51.35 \%)$ & \\
\hline
\end{tabular}

Analysis using independent student's t-test, Chi square or Mann Whitney $\mathrm{U}$ test as appropriate

Data are presented as median (interquartile range, IQR), number (\%) or mean \pm standard deviation as appropriate.

II Indicates statistical significance.

Table 2 illustrates studied neonatal outcomes showing no significant difference regarding neonatal weight, neonatal sex distribution, Apgar score at $1^{\text {st }}$ and $5^{\text {th }}$ minutes, as well as mean umbilical artery pCO2, p02 and mean umbilical vein $\mathrm{pH}(P>0.05)$.
However, umbilical artery base deficit was significantly more and $\mathrm{pH}$ was significantly less in case group $(P=0.015 ; P=0.029$; respectively).

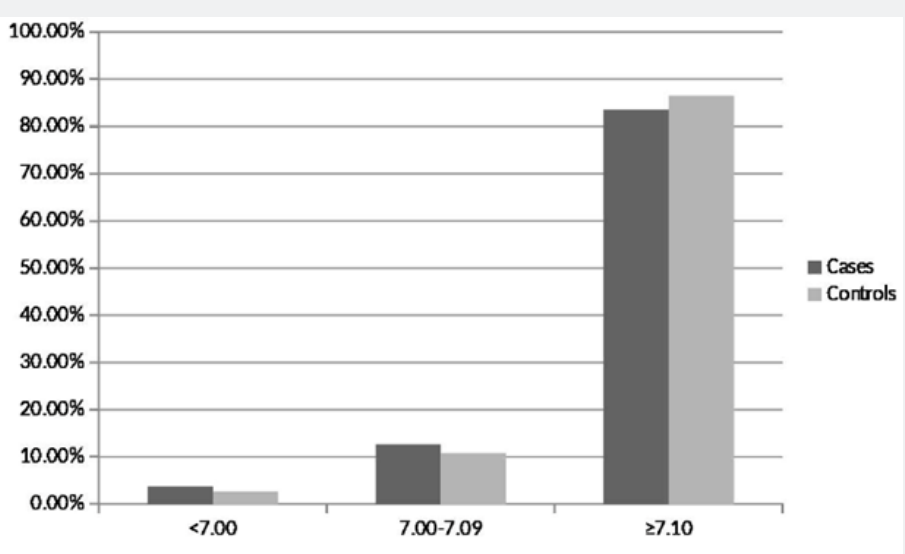

Figure 1: Apgar scores at 5th minute among studied groups.

Umbilical artery $\mathrm{pH}$ Values

Fisher exact test, $\mathrm{P}=0.281$

Relative Risk: $\mathrm{RR}=1.218,95 \% \mathrm{Cl}$ (0.5689 to 2.6064$), \mathrm{p}=0.612$

Odd Ratio: $\mathrm{OR}=1.261,95 \% \mathrm{Cl}(0.5160$ to 3.0797$), \mathrm{p}=0.611$

Interestingly, Figure 1 illustrates no significant difference between cases and controls regarding number of neonates with arterial $\mathrm{pH}$ less than 7.1 (13 (16.5\%) versus 10 (13.5\%); respectively) ( $\mathrm{RR}=1.218, \mathrm{P}=0.612 ; \mathrm{OR}=1.261, \mathrm{P}=0.611 ; 95 \% \mathrm{CI}$ ), while Figure 2 shows that number of neonates with significant impairment of arterial base deficit (>8mmol/L) didn't show significant difference between cases and controls (16(20.3\%) versus 11(14.9\%); respectively) $(\mathrm{RR}=1.363, \mathrm{P}=0.386$; $\mathrm{OR}=1.455$, $\mathrm{P}=0.384 ; 95 \% \mathrm{CI})$. 


\section{Journal of Gynecology and Women's Health}

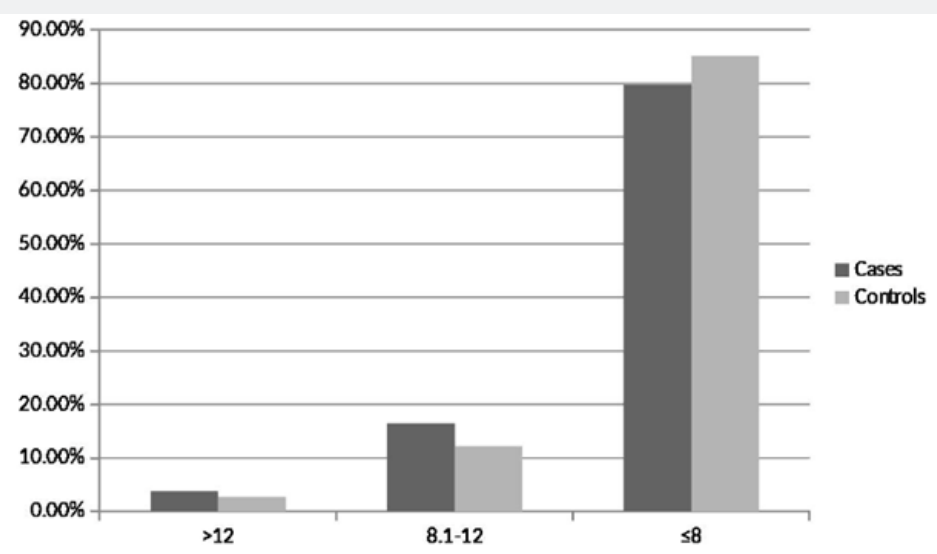

Figure 2: Umbilical artery $\mathrm{pH}$ ranges among studied groups.

Umbilical artery base deficit values in millimoles /litre

Fisher exact, $p=0.682$

Relative Risk: $\mathrm{RR}=1.363,95 \% \mathrm{Cl}(0.6772$ to 2.7413$), \mathrm{P}=0.386$

Odd Ratio: $\mathrm{OR}=1.455,95 \% \mathrm{Cl}(0.6258$ to 3.3807$), \mathrm{P}=0.384$

Table 3: Correlation between demographic characteristics, neonatal sex, neonatal weight, and studied neonatal outcomes among all participants.

\begin{tabular}{|c|c|c|c|c|c|c|c|c|}
\hline \multirow{2}{*}{} & \multicolumn{2}{|c|}{ Apgar 1 } & \multicolumn{2}{c|}{ Apgar 5 } & \multicolumn{2}{c|}{ Umbilical Artery PH } & \multicolumn{2}{c|}{ Umbilical Artery B.D. } \\
\cline { 2 - 10 } & R & P Value & R & P Value & R & P Value & R & P Value \\
\hline Age & 0.093 & 0.415 & 0.038 & 0.741 & 0.099 & 0.385 & 0.006 & 0.96 \\
\hline Parity & 0.19 & 0.094 & 0.152 & 0.181 & 0.11 & 0.332 & 0.133 & 0.243 \\
\hline Gestational age & 0.147 & 0.195 & 0.158 & 0.165 & 0.126 & 0.267 & 0.07 & 0.54 \\
\hline Neonatal sex & 0.012 & 0.919 & 0.084 & 0.463 & 0.115 & 0.312 & -0.009 & 0.938 \\
\hline Neonatal weight & 0.034 & 0.098 & 0.102 & 0.287 & 0.276 & 0.781 & -0.021 & 0.729 \\
\hline
\end{tabular}

Apgar 1, Apgar score at 1st minute; Apgar 5, Apgar score at 5th minute; B.D., base deficit; r, Pearson's correlation coefficient Analysis using Pearson's correlation test.

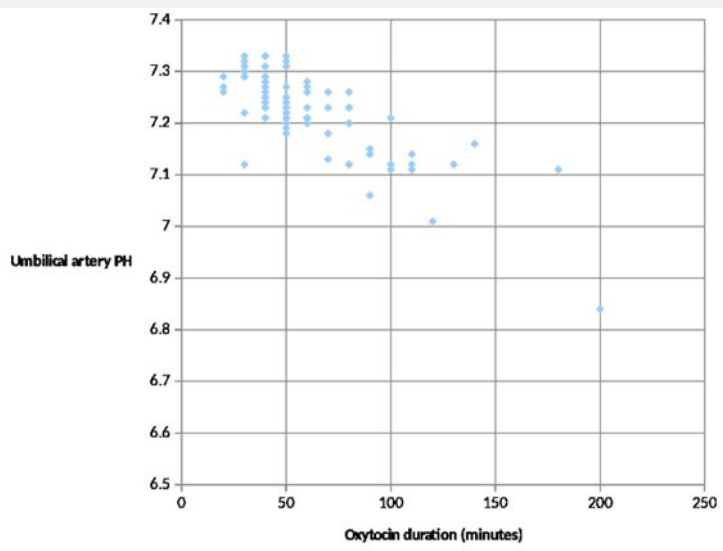

Figure 3: Correlation between duration of oxytocin administration and umbilical artery pH among case group.

$r$ (Pearson's correlations coefficient): $-0.789, P<0.001$ 
In this study, Apgar scores at $1^{\text {st }}$ and $5^{\text {th }}$ minute were not correlated to any of maternal age, parity, gestational age, neonatal sex or neonatal weight. Also, umbilical artery $\mathrm{pH}$ and base excess have non-significant correlations to these variables $(P>0.05$; Table $3)$. Figure 3 illustrates Pearson's correlation test between duration of oxytocin administration and umbilical artery $\mathrm{pH}$ among group I, showing a significant negative correlation $(P<0.001)$.

\section{Discussion}

Although labor augmentation using oxytocin has been investigated in few trials, most of these trials have focused on various outcomes mainly duration of labor, rate of Cesarean sections or instrumental deliveries and maternal experience with few of them [9-11], have measured neonatal outcomes as a primary outcome.

The current study reported that low-dose oxytocin use is associated with reduction of neonatal umbilical artery $\mathrm{pH}$ and increase in base deficit without compromising the neonatal clinical condition or affecting Apgar scores.

Oxytocin transiently compromises fetal circulation by increasing duration, frequency and strength of uterine contractions where this effect was studied by Olofsson et al. [12], through ultrasound and Doppler assessment where uterine and umbilical artery flow resistance increased significantly during uterine contractions reflecting rapid and exaggerated increase of vascular resistance in both arteries.

The biochemical disturbance must be significant before Apgar score is affected [13]. Moreover, a number of factors may influence Apgar score, including trauma, drugs, infections, congenital anomalies, hypovolemia, hypoxia and preterm birth. Accordingly, it is inappropriate to use an Apgar score alone to establish the diagnosis of asphyxia [14].

In the year 2008, oxytocin was added to the list of medications designated as high alert by the Institute for Safe Medication, USA [15]; such drugs are defined as those bearing a heightened risk of harm when used in error. Not following the guidelines regarding use of oxytocin is probably one reason for adverse neonatal outcomes. The use of oxytocin is on the increase and it is estimated that about $50 \%$ of nulliparous and $20 \%$ of parous women receive oxytocin during delivery [16]. A recent Cochrane review by Kenyon et al. involved 4 randomized trials and found no significant difference for Apgar scores, umbilical cord blood gases, admission to special care baby unit, or neonatal mortality between cases managed by high-dose and those managed by lowdose oxytocin for labor augmentation [17].

The current study found no statistical difference neither in Apgar scores at $1^{\text {st }}$ and $5^{\text {th }}$ minutes nor in distribution of Apgar score values between both groups, where 5 neonates $(6.3 \%)$ of the case group and 4 neonates (5.4\%) of the control group had a score less than 7 at $1^{\text {st }}$ minute while one neonate of each group had a score less than 7 at $5^{\text {th }}$ minute. No neonatal deaths were reported during the study. Three Neonates were diagnosed with TTN and no special care admissions occurred for any of the neonates involved.

These results are comparable to those of Chan and To, in their prospective study using low-dose oxytocin, who found that $6 \%$ of the neonates of the study group had Apgar scores less than 7 at one minute, while $100 \%$ of the neonates of their study group had Apgar scores more than 7 at 5 minutes [18]. Also, our results agree with Merrill and Zaltnik (3.9\% of the studied neonates had Apgar score $<7$ at 5 minutes) [19]. Melin et al. [20] have classified neonatal acidemia according to umbilical artery $\mathrm{pH}$ at birth as mild or pre-acidemia at 7.10 to 7.19 , moderate acidemia at 7.00 to 7.09 , and severe acidemia at $\mathrm{pH}$ less than 7.00. In a similar context, and for the purpose of prediction of the likelihood of neonatal encephalopathy, Low et al. [21] have classified neonatal acidosis, as regards umbilical arterial base deficits, as mild; 4-8 $\mathrm{mmol} / \mathrm{L}$, moderate; 8-12mmol/L, and severe acidosis; greater than $12 \mathrm{mmol} / \mathrm{L}$. Accordingly, in the current study, numbers of neonates with $\mathrm{pH}<7.1$ and those with base deficits $>8 \mathrm{mmol} /$ Litre, were comparable between both groups with no significant difference ( $O R=1.261$ and 1.455; respectively), while, surprisingly, the case group showed a significantly lower arterial $\mathrm{pH}$ and a significantly higher arterial base deficit as compared to controls, which emphasizes the undeniable effect of oxytocin on the arterial acid-base status but this effect was not enough to increase the risk of developing neonatal academia, which agrees with Merrill and Zaltnik in their randomized double masked trial [19]. Also, Thorp et al. [10] conducted a study on 1423 primigravid women where $43 \%$ of them received high-dose oxytocin augmentation and no significant difference was noticed in mean arterial $\mathrm{pH}$ between oxytocin and no oxytocin groups. Contradictorily, Oscarsson et al. [16] in their massive retrospective study on 106,755 deliveries, concluded that oxytocin use increased the risk of low Apgar score at $5^{\text {th }}$ minute and neonatal special care admission but their study remains limited by being retrospective and hence subject to recall bias as well as heterogeneity of population recruited from 10 different hospitals and not considering factors like uterine hyperstimulation.

In the current study, fifteen umbilical artery $\mathrm{pH}$ values (20.3\%) of the control group were less than 7.2 which agrees with those of Akoury et al. [9] (20\%) but they have used base deficit of $7 \mathrm{mmol} / \mathrm{L}$ as a cut-off to diagnose neonatal acidosis and hence, $29 \%$ of cases and $27 \%$ of controls in their study were acidotic. Different modes of delivery might be another reason for different results.

The fact of occurrence of acidemia in non-augmented labors was explained by Yoon and Kim who concluded a normal significant fall in umbilical artery $\mathrm{pH}$ and bicarbonate with the presence of labor and increased duration of second stage of labor in healthy term neonates [22]. The current study hasn't considered neonates with mild acidemia/acidosis as it is thought that mild acidosis occurs in almost all normal labors [23]. 
The current study uniquely reported the significant negative correlation between duration of oxytocin administration among the case group and umbilical artery $\mathrm{pH}$ which signifies the compromising effect of oxytocin on neonatal circulation studied by Olofsson et al. [12] and correlates with the duration of use not only the dose which has been studied in few trials. As oxytocin impairs the utero-placental and umbilical circulations, there might be a gradual accumulation of lactates or gradual affection of the fetal ability to compensate and wash lactates out of the circulation even in absence of uterine hyper-stimulation.

The limitation of this study is that it hasn't studied the incidence of meconium staining at birth and risk of meconium aspiration as a neonatal outcome. Also, the progress of labor hasn't been correlated with the results. Moreover, only low-dose oxytocin was studied and further studies are needed to evaluate effect of highdose oxytocin compared to controls and emphasize the effect of duration of oxytocin use on neonatal outcome with larger number of patients and correlate it to other neonatal parameters.

\section{Conflict of Interest}

No actual or potential conflict of interest in relation to this manuscript exists.

\section{References}

1. Zhu BP, Grigorescu V, Le T, Lin M, Copeland G, et al. (2006) Labor dystocia and its association with inter-pregnancy interval. AJOG 195(1): 121-128.

2. O'Driscoll K, Foley M, MacDonald D (1984) Active management of labor as an alternative to cesarean section for dystocia. Obstet Gynecol 63(4): 485-490.

3. Kupittayanant S, Luckas MJM, Wray S (2002) Effects of inhibitng the sarcoplasmic reticulum on spontaneous and oxytocin-induced contractions of human myometrium. BJOG 109(3): 289-296.

4. Wray S, Jones K, Kupittayanant S, Li Y, Matthew A, et al. (2003) Calcium signaling and uterine contractility. J Soc Gynecol Investig 10(5): 252264

5. Gilstrap LC (2004) Fetal acid-base balance. In: ( $5^{\text {th }}$ edn),Maternal-Fetal Medicine. Philadelphia, PA Saunders, USA, p. 23.

6. Hayes EJ, Weinstein L (2008) Improving patient safety and uniformity of care by a standardized regimen for the use of oxytocin. AJOG 198(6): 622

7. American College of Obstetricians and Gynecologists (2009) ACOG Practice Bulletin No. 106 Intrapartum fetal heart rate monitoring: nomenclature, interpretation, and general management principles. Obstet Gynecol 114(1): 192-202.
8. ACOG Committee on Practice Bulletins - Obstetrics (2009) ACOG Practice Bulletin No. 107: Induction of labor. Obstet Gynecol 114(2 Pt 1): 386-397.

9. Akoury H, MacDonald F, Brodie G, Caddick R, Chaudhry N, et al. (1991) Oxytocin augmentation of labor and neonatal outcome in nulliparas. Obstet Gynecol 78(2): 227-230.

10. Thorp JA, Boylan PC, Parisi VM, Heslin EP (1988) Effects of highdose oxytocin augmentation on umbilical cord blood gas values in primigravid women. Am J Obstet Gynecol 159(3): 670-675.

11. Simpson KR, James DC (2008) Effects of oxytocin-induced uterine hyper-stimulation during labor on fetal oxygen status and fetal heart rate patterns. Am J Obstet Gynecol 199(1): 34.

12. Olofsson P, Thuring-Jönsson A, Marsál K (1996) Uterine and umbilical circulation during the oxytocin challenge test. Ultrasound Obstet Gynecol 8(4): 247-251.

13. Papile LA (2001) The Apgar score in the $21^{\text {st }}$ century. $N$ Engl J Med 344(7): 519-520.

14. American Academy of Pediatrics (AAP) and American College of Obstetricians and Gynecologists (ACOG) (2006) The Apgar scores. Pediatrics 117: 4.

15. Institute for Safe Medical Practices (2008) ISMP High alert medications, USA.

16. Oscarsson ME, Amer-Wahlin I, Rydhstroem H, Kallen K (2006) Outcome in obstetric care related to oxytocin use. A population-based study. Acta Obstet Gynecol Scand 85(9): 1094-1098.

17. Kenyon S, Tokumasu H, Dowswell T, Pledge D, Mori R, et al. (2013) High-dose versus low-dose oxytocin for augmentation of delayed labour. Cochrane Database Syst Rev 7: CD007201.

18. Chan S, To W (2009) Correlation between intrapartum cardiotocogram findings and cord blood $\mathrm{pH}$ in term and preterm labors. HKJGOM 9(1): 321-326.

19. Merrill D, Zaltnik F (1999) Randomized, double-masked comparison of oxytocin dosage in induction and augmentation of labor. Obstet Gynecol 94(3): 455-463.

20. Melin M, Bonnevier A, Cardell M, Hogan L, Herbst A, et al. (2008) Changes in the ST-interval segment of the fetal electrocardiogram in relation to acid-base status at birth. BJOG 115(13): 1669-1675.

21. Low JA, Lindsay BG, Derrick EJ (1997) Threshold of metabolic acidosis associated with newborn complications. AJOG 177(6): 1391-1394.

22. Yoon BH, Kim SW (1994) The effect of labor on the normal values of umbilical blood acid-base status. Acta Obstet Gynecol Scand 73(7): 555-561.

23. Ross MG, Gala R (2002) Use of umbilical artery base excess: Algorithm for the timing of hypoxic injury. AJOG 187(1): 1-9. 

(C) Commons Attribution 4.0 License BY DOI: 10.19080/JGWH.2020.18.555977

\section{Your next submission with Juniper Publishers} will reach you the below assets

- Quality Editorial service

- Swift Peer Review

- Reprints availability

- E-prints Service

- Manuscript Podcast for convenient understanding

- Global attainment for your research

- Manuscript accessibility in different formats

( Pdf, E-pub, Full Tsext, Audio)

- Unceasing customer service

Track the below URL for one-step submission https://juniperpublishers.com/online-submission.php 